\title{
An efficient tie-line ATC calculation method with N-1 contingencies
}

\author{
Yuta Ohsaki ${ }^{\mathrm{a}}$, Masashi Hitosugi ${ }^{\mathrm{a}}$, Shinichi Iwamoto ${ }^{\mathrm{a}^{*}}$, \\ Hideo Hosogoe $^{\mathrm{b}}$, Mitsuhiro Matsumoto ${ }^{\mathrm{b}}$ \\ ${ }^{a}$ Waseda University, 3-4-1 Ohkubo Shinjuku, Tokyo 169-8555, Japan \\ ${ }^{b}$ Tohoku Electric Power Company,1-7-1 Aoba Sendai, Miyagi 980-8550, Japan
}

\begin{abstract}
Recently, in the process of deregulation, the fast evaluation of accurate ATC (available transfer capability) of tie-lines has become a more important problem from the viewpoint of the effective use of networks. Therefore, we propose a smart efficient calculation method for ATC with transient stability constraints. In this paper, first we focus our attention on characteristics of ATC and reduce the calculation time drastically by it. Second, we propose a new index for finding critical cases quickly using a transient energy function, and also propose a smart efficient ATC calculation method by using the relationship between an increment of generator outputs and CCTs (critical clearing times). CCT is calculated by the trial and error method. Finally, we compare an estimated ATC with ATC at the tie-line fault case. We confirm the validity of the proposed method by running simulations using an IEEJ EAST 10 machine 47 bus system.
\end{abstract}

Keywords: Power system, transient stability, available transfer capability, transient energy, critical clearing time

\section{Introduction}

In Japan, power industry deregulation has progressed and power transactions between two areas using tie-lines have been increasing. However, because the number of Japanese power systems consisting of 10 areas has grown for the purpose of adjusting supply and demand in their own areas, the tie-lines do not have the enough capacity margin to transmit the required additional power. Therefore, fast and accurate evaluation of the tie-line ATC has been more important from the point of effective use of networks.

The tie-line ATC means the critical transmission power which we can add to the present power flow of a tie-line. Here in Japan "critical transmission power" is defined as the maximum level of additional transmission power that can be operated safely when we consider all conditions (all plausible contingencies and all states of power flows). Although there are thermal capacity constraints, frequency constraints, voltage constraints and transient stability constraints as the constraints which determine the value of the tie-line ATC, in this paper, we only consider the transient stability constraints. Generally, when we calculate the value of the tie-line ATC considering the transient stability constraints, we judge whether a case is stable for all contingencies, increasing the power flow of the tie-line gradually from the base power flow, and finally, we determine the value of the critical transmission power of the tie-line. Therefore, because we must carry out the transient stability calculations for all combinations of contingencies and states of power flow, we need a large amount of calculation times. Therefore, we propose a smart approach which can calculate the tie-line ATC efficiently. Simulations are carried out using the IEEJ EAST 10 machine 47 bus system and we can then confirm the validity of the proposed approach.

\footnotetext{
* Manuscript received May 15, 2013; revised July 29, 2013.

Corresponding author. Tel.: +81 3-5286-3184; E-mail address: iwamoto.lab.pwrs@gmail.com.
} 


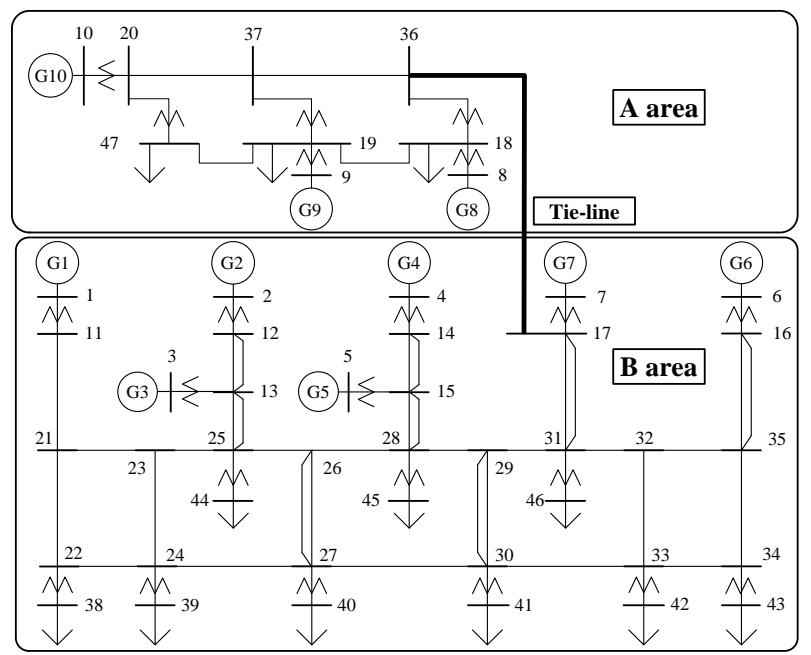

Fig. 1. EAST 10 machine 47 bus system [7]

\section{Calculation of Tie-Line ATC}

\subsection{Simulations}

In our simulations, we use the IEEJ EAST 10 machine 47 bus system shown in Fig. 1. In this paper we divide the system into Areas A and B as depicted in Fig. 1 and consider the transmission line which connects the two areas as the tie-line. Also, we take into account some of the assumptions for simplicity as follows:

A) Change of power flow of the tie-line is carried out by increasing the output of a certain generator in Area A and decreasing the output of a certain generator in Area B, except for slack generator G3 in each case. Therefore, all together we have ( 3 machines $\times 6$ machines $=18$ combinations).

B) Three phase to ground faults occur at buses.

(Fault clearing is done by one circuit line opening of a double circuit line.)

C) The simulation time is 4 [s]. The fault clearing time is 0.1 [s] considering the actual speed of circuit breakers. The criterion for stepping-out is \pm 180 [deg] for the center of the inertia (COI) frame.

\section{Characteristics of ATC}

\subsection{Influence of output-decreasing generators on ATC[1]}

In this section, we explain the characteristics of ATC. The next three conditions can be considered as having effects on the values of ATC; faulted buses, output increasing generators (OIGs) and output decreasing generators (ODGs).

For example, the range of ATC (the difference between maximum and minimum values of ATCs) can be determined when we change only the faulted buses with a combination of one OIG and one ODG. As there are many combinations of one OIG and one ODG, we consider the average value of the ATC range as an influence of the faulted buses on ATC.

In Table 1, we summarize the influence of these three conditions on ATC only for the critical cases (top 10\% of the critical rankings). From Table 1, we can find that the influence of faulted buses and OIGs increases, while the influence of ODGs decreases in critical cases. In other words, in critical cases, the influences of faulted buses and OIGs are dominant, and the influence of ODGs is insignificant.

From the above results we can think of the following screening method. If ODGs do not affect the value of ATC, a method for finding the critical cases must be effective, which decreases the outputs from all generators in Area B equally, and not only from the generator. Because there are 6 ODGs in Area B, we can reduce the calculation time to $1 / 6$ th using this method. 
Table 1. Influence of three conditions on ATC (for critical cases)

\begin{tabular}{c|c}
\hline \hline & Range of ATC (average) \\
\hline Selection of faulted buses & $4.28[\mathrm{pu}]$ \\
\hline Selection of output increasing generators & $4.63[\mathrm{pu}]$ \\
\hline Selection of output decreasing generators & $0.15[\mathrm{pu}]$ \\
\hline
\end{tabular}

Table 2. Ranking of ATC

(a) Output decrease of one GEN

(b) Output decrease of all GEN

\begin{tabular}{|c|c|c|c|c|c|}
\hline Ranking & case & ATC[pu] & Ranking & case & ATC[pu] \\
\hline 1 & G10-f36-o17 & 0.70 & - 1 & G10-f36-o17 & 0.76 \\
\hline 1 & G9-f36-o17 & 0.70 & - 1 & G9-f36-o17 & 0.76 \\
\hline 3 & G8-f36-o17 & 0.72 & - 3 & G8-f36-o17 & 0.79 \\
\hline 4 & G10-f37-o36 & 0.85 & 4 & G10-f20-o37 & 0.90 \\
\hline 5 & G10-f20-o37 & 0.89 & 5 & G10-f37-o36 & 0.93 \\
\hline 6 & G9-f37-o36 & 0.90 & - & G9-f37-o36 & 1.00 \\
\hline 7 & G10-f37-o20 & 1.07 & - & G10-f37-o20 & 1.14 \\
\hline 8 & G10-f36-o37 & 1.10 & -1 & G10-f36-o37 & 1.20 \\
\hline 9 & G9-f19-o18 & 1.15 & $-\quad 9$ & G9-f19-o18 & 1.22 \\
\hline 10 & G9-f36-o37 & 1.16 & 10 & G9-f17-o36 & 1.24 \\
\hline
\end{tabular}

G $\square$-f $\square$-o $\square$ G $\square$ :Output Increasing Generator f $\square$ :Fault Bus $\quad$ o $\square$ :Other Bus

Table 3. Total number of output increasing generators and tie-line ATC

(a)With the tie-line fault case

\begin{tabular}{c|c}
\hline Number of OIGs & ATC[pu] \\
\hline 1 & $0.76 / \mathrm{G} 10$-fault36-other17 \\
\hline 2 & $0.79 / \mathrm{G} 8,9$-fault36-other17 \\
\hline 3 & $0.79 / \mathrm{G} 8,9,10$-fault36-other17 \\
\hline
\end{tabular}

(b)Without the tie-line fault case

\begin{tabular}{c|c}
\hline Number of OIGs & ATC[pu] \\
\hline 1 & $0.90 / \mathrm{G} 10$-fault20-other37 \\
\hline 2 & $1.00 / \mathrm{G} 9,10$-fault37-other36 \\
\hline 3 & $1.36 / \mathrm{G} 8,9,10$-fault37-other36 \\
\hline
\end{tabular}

(OIGs: Output Increasing Generators)

Table 2 compares the ATC ranking when outputs of individual generators are decreased with the ATC ranking when outputs of all generators in $\mathrm{B}$ area are decreased equally. From the two critical rankings in Table 2, we can see that the ranking of (B) is in good agreement with the ranking of (A). From the above results, we can confirm the effectiveness of this method. In this paper, we use this method for calculating ATC hereafter.

\subsection{Number of output increasing generator}

In this paper, we have assumed that output of only one generator in A area is increased for change of the power flow of the tie-line. However, in reality, it is only necessary to change the output of one generator for calculating the tie-line ATC. This is because power systems become unstable if any of generators steps out and a generator often steps out when the output of the one generator is increased.

Table 3 shows the relationship between the total number of OIGs and the tie-line ATCs. (The tie-line ATCs are calculated with increasing outputs of OIGs equally.) As shown in Table3, the tie-line ATCs become larger as the number of OIGs increase. From the above results, we can confirm that it is adequate to take into account of the cases in which output of one OIG is increased, for calculating the tie-line ATC. 


\subsection{Consideration of the fault case}

When we calculate the transient stability ATC, if the most critical cases can be judged before the simulations, we can reduce the number of calculations. Table 4 shows the top ten cases of ATC rankings of generator combinations at the three kinds of load multipliers (90\%). The highlighted parts in the table mean faults at the tie-line buses.

From Table 4, we believe that the faults at the A area are the near critical cases. This is because the transmission lines in the A area are heavy-loaded. Also, we can see that the fault case at the tie line is very severe case. However, we focus on the fault cases without tie line fault. Then, we compare the tie line fault case with other fault cases and finally decide tie-line ATC.

If we focus on fault cases without tie line fault, we can find that the faults at buses near outputincreasing generators are critical cases. From these results, we can generate the following screening method. Even if a screening object is limited to a fault at buses near output-increasing generators, we can calculate the transient stability ATC accurately and reduce the calculation time. Assuming that there are $\mathrm{N}$ faults, we can reduce the calculation time to $1 / \mathrm{N}$ by using this method.

Table 4. ATC ranking of generator combinations

\begin{tabular}{|c|c|c|c|c|c|c|c|c|c|}
\hline \multirow{2}{*}{ rank } & \multicolumn{3}{|c|}{ G8_up-Gall_down } & \multicolumn{2}{c|}{ G9_up-Gall_down } & \multicolumn{3}{|c|}{ G10_up-Gall_down } \\
\cline { 2 - 11 } & fault & other & ATC & fault & other & ATC & fault & other & ATC \\
\hline 1 & 36 & 17 & 0.79 & 36 & 17 & 0.76 & 36 & 17 & 0.76 \\
\hline 2 & 17 & 36 & 1.34 & 37 & 36 & 1.00 & 20 & 37 & 0.90 \\
\hline 3 & 37 & 36 & 2.59 & 19 & 18 & 1.22 & 37 & 36 & 0.93 \\
\hline 4 & 18 & 19 & 2.65 & 17 & 36 & 1.26 & 37 & 20 & 1.14 \\
\hline 5 & 36 & 37 & 2.70 & 36 & 37 & 1.27 & 36 & 37 & 1.20 \\
\hline 6 & 19 & 18 & 3.28 & 19 & 47 & 1.41 & 17 & 36 & 1.24 \\
\hline 7 & 37 & 20 & 3.35 & 37 & 20 & 1.48 & 19 & 18 & 1.36 \\
\hline 8 & 20 & 37 & 3.42 & 20 & 37 & 2.24 & 19 & 47 & 1.52 \\
\hline 9 & 19 & 47 & 3.45 & 18 & 19 & 2.31 & 47 & 19 & 1.82 \\
\hline 10 & 25 & 26 & 3.99 & 25 & 26 & 2.96 & 18 & 19 & 2.23 \\
\hline
\end{tabular}

\section{Relationship of CCT and Generator Output}

\subsection{Relationship between $\Delta$ generator output and CCT}

Generally, CCT is considered as the index for assessing the degree of system stability (unstability) for the contingency.

For instance, if a CCT at a contingency is larger than an actual circuit breaker operating time in the power system, the transient stability in the power systems is stable, otherwise it is unstable.

Fig. 2 shows the change of CCT when the generator output increases.

Here, Fig. 2 shows the result of fault cases at the bus near output-increasing generator 10. Similar linear relationships were also obtained for other cases. With the results, we propose an ATC calculation method as follows.

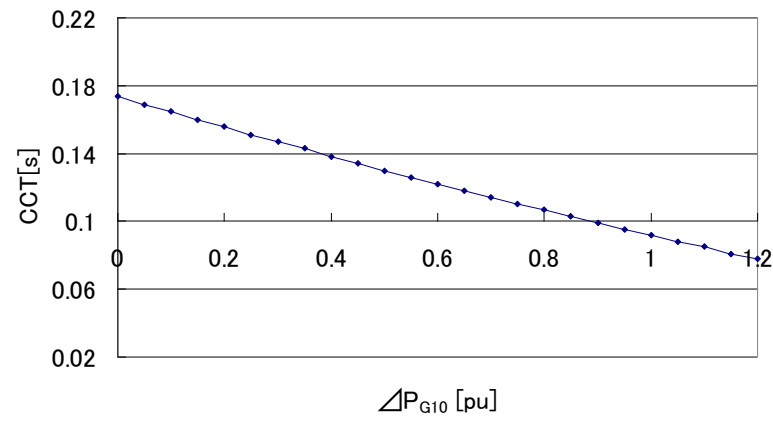

Fig. 2. Generator output -CCT relationship (fault 20-37). 


\subsection{ATC calculation method by using CCT}

Step 1: Compute CCTpresent at the present state (The increment of tie-line power flow $\Delta \mathrm{Ptie}=0[\mathrm{pu}]$ ) by using the RIDGE theory.

Step 2: Compute CCTfuture at a future state (For example, $\Delta$ Ptie $=0.5[\mathrm{pu}]$ ) by using the RIDGE theory.

Step 3: Calculate the linear expression between the points computed above (CCTpresent and CCTfuture). Calculate the crossing point between the assumed circuit breaker operating time $(0.10[\mathrm{~s}])$ in the power system and the linear expression. The crossing point (tie-line power flow) is an ATCestimate.

$$
A T C_{\text {estimate }}=\frac{\Delta \text { Ptie } \times\left(0.1-C C T_{\text {present }}\right)}{C C T_{\text {future }}-C C T_{\text {present }}}
$$

Repeat the ATC estimation to improve the accuracy for $\mathrm{ATC}_{\text {estimate. }}$

Step 4: Compute CCT by setting the tie-line flow increment equal to the estimated ATC. When CCT equals approximately $0.1[\mathrm{~s}]$, finish the calculation.

Step 5: Compute CCT by using the RIDGE theory. Then, calculate a newly estimated ATC by using two points.

The accuracy of the estimated ATC will be improved as the reiteration number increases. Considering the calculation time, we set only one reiteration.

\section{Screening Method}

\subsection{Severity index using the individual TEF (proposed index) [6]}

Multiplying (2) by $\omega_{i}$ and then integrating with respect to time, we can obtain an individual transient energy function (TEF) as shown in (2).

$$
\begin{aligned}
V_{i} & =\int_{0}^{t}\left(M_{i} \frac{d \omega_{i}}{d t}-P_{m i}+P_{e i}\left(Y^{P}, \theta\right)+R_{i} P_{C O A}\right) \cdot \omega_{i} d t \\
& =\int_{0}^{\omega_{i}}\left(M_{i} \omega_{i}\right) d \omega_{i}+\int_{\theta_{i}^{s}}^{\theta_{i}}\left(-P_{m i}+P_{e i}\left(Y^{P}, \theta\right)+R_{i} P_{C O A}\right) d \theta_{i} \\
& =\frac{1}{2} M_{i} \omega_{i}^{2}+\int_{\theta_{i}^{s}}^{\theta_{i}}\left(-P m_{i}+P e_{i}\left(Y^{P}, \theta\right)+R_{i} P_{C O A}\right) d \theta_{i} \\
& =V_{k i}+V_{p i}
\end{aligned}
$$

where $V_{p i}$ is individual potential energy, $Y^{P}$ is post fault admittance matrix, and $V_{k i}$ is individual kinetic energy

In this paper, we first make a severity index using the individual TEF and propose a method which estimates the severest fault point by using the index. The proposed index is shown in equation (3).

Proposed_index $=\left(V_{i u p}^{1 p u}\left(T_{c l}\right)-V_{\text {iup }}^{\text {base }}\left(T_{c l}\right)\right) \times \theta_{i u p}^{1 p u}\left(T_{c l}\right)$

$V_{u p} \quad:$ the value of individual TEF of output increasing generators

1pu : the value when output is increased by $1 \mathrm{pu}$

base : the value with base output

$\left(T_{c l}\right)$ : the fault clearing time $(0.1 \mathrm{sec})$

We use the value of the individual TEF when the output is increased by $1 \mathrm{pu}$ in order to estimate the influence of the output increment. The physical meaning of the index is that we take into account the effects of the individual TEF and the rotor angle in the form of multiplication. When we consider the tieline ATC with transient stability constraints, we must consider "the influence of the output increment", 
"the severity of the fault" and "the severity of the post-fault condition" together.

Also, we use the value of the individual TEF at $T_{c 1}$ in (3). This is because the value of the individual TEF does not change after the $T_{c \mathrm{l}}$ based on the characteristics of the individual TEF.

\section{The Proposed Method of The Tie Line ATC}

The outline of the proposed method is as follows:

1) First, we estimate the most critical cases from the faults at buses near output-increasing generators by using the individual transient energy functions. Then, we calculate the ATC value of the estimated case using the linear relationship of the increment of the generator outputs and CCTs.

2) There is no guarantee that the case estimated in Step (1) is the most critical case that determines the value of the tie-line ATC (Without the tie-line fault case). Thus, for calculating an accurate tie-line ATC (Without the tie-line fault case), we carry out the transient stability calculations for all the cases without the tie-line fault case under the condition where the power flow of the tie-lines is increased to the estimated value of the tie-line ATC.

3) If some unstable cases exist in the transient stability calculations for the cases, we estimate the most critical case out of the unstable cases using the values of the stepping-out times, and calculate the ATC value for the estimated case.

4) Next, we carry out transient stability calculations for the unstable cases again under the condition where the power flow of the tie-lines is increased to the estimated value of the tie-line ATC.

5) After repeating these Steps, we can locate the most critical case, and the ATC of that case becomes the tie-line ATC (Without the tie-line fault case). Then, we compare it with ATC at the tie-line fault case, and finally locate the tie-line ATC.

\section{Simulations}

\subsection{Simulation}

Simulations are carried out to verify the validity of the proposed ATC calculation method using the IEEJ 10 machine 47 bus model system in Fig. 1 [7]. The load multiplier is $90 \%$ for Simulation.

1) First, we estimate the most critical case from the faults at the buses near output-increasing generators by using the transient energy functions. Table 5 shows the results of the screenings. We calculate the ATC value of the estimated case using the proposed method. Table 6 shows the transient stability ATC (candidate).

2) There is no guarantee that the case estimated in step 1) is the most critical case that determines the value of the tie-line ATC (without the tie-line fault case). Thus, for calculating an accurate tie-line ATC (without the tie-line fault case), we carry out transient stability calculations for all the cases without the tie-line fault case under the condition where the power flow of the tie-lines is increased to the estimated value of the tie-line ATC $(0.89 \mathrm{pu})$.

3) As a result, the all cases without the tie-line fault case are stable. Therefore, we calculate the ATC at the tie-line fault case using the proposed method. Table 7 shows the transient stability ATC at the tie-line fault case.

From Table 6 and 7, we compare the ATC $(0.77 \mathrm{pu})$ with the other fault cases ATC $(0.89 \mathrm{pu})$, and we can see the tie-line fault case is the most critical case. Therefore, $0.77 \mathrm{pu}$ (calculated at step 2)) indicates the real tie-line ATC. Finally, we show the accuracy of the proposed method in Table 8.

We can confirm that the proposed method is effective for calculating the real tie-line ATC for N-1 contingencies fast and accurately.

Table 5. Result of screenings

\begin{tabular}{|c|c|c|c|}
\hline Case & $\begin{array}{c}\text { G10_up } \\
\text { Gall down }\end{array}$ & $\begin{array}{c}\text { G9_up } \\
\text { Gall down }\end{array}$ & $\begin{array}{c}\text { G8_up } \\
\text { Gall down }\end{array}$ \\
\hline fault-other & fault20-other37 & fault19-other18 & fault18-other19 \\
\hline Index_Value & 229.066 & 198.899 & 144.777 \\
\hline
\end{tabular}


Table 6. Transient stability ATC (candidate)

\begin{tabular}{|c|c|}
\hline case & $\begin{array}{c}\text { G10_up-Gall_down } \\
\text { fault20-other37 }\end{array}$ \\
\hline ATC[pu] & 0.89 \\
\hline
\end{tabular}

Table 7. Transient stability ATC at the tie-line fault case

\begin{tabular}{|c|c|}
\hline case & $\begin{array}{c}\text { G10_up-Gall_down } \\
\text { fault36-other17 }\end{array}$ \\
\hline ATC[pu] & 0.77 \\
\hline
\end{tabular}

Table 8. Accuracy of the proposed method

\begin{tabular}{|c|c|c|}
\hline case & $\begin{array}{c}\text { ATC } \\
\text { (TrueValue) }\end{array}$ & $\begin{array}{c}\text { ATC } \\
\text { (Proposed Method) }\end{array}$ \\
\hline $\begin{array}{c}\text { G10_up-Gall_down } \\
\text { fault20-other37 }\end{array}$ & 0.76 & 0.77 \\
\hline $\begin{array}{c}\text { G10_up-Gall_down } \\
\text { fault36-other17 }\end{array}$ & 0.90 & 0.89 \\
\hline
\end{tabular}

\section{Conclusion}

In this paper, we first explained the characteristics of ATC that are affected by the faulted buses and OIGs that are influential, and also described that the influence of ODGs is insignificant in critical cases. Next, we reduced the combinations of the generators to $1 / 6$ th by decreasing the outputs of all the generators equally in Area B.

Secondly, we proposed a smart and effective screening method which estimates critical cases by using the proposed index which consists of individual TEF. Next, we also proposed the index that estimates the critical case using the individual transient stability and the ATC calculation method using the linear relationship between the increments of generator outputs and CCTs.

The validity of the proposed methods has been confirmed by running simulations for the IEEJ EAST 10 machine 47 bus system. From the above results, it can be considered that the proposed approach is a smart and effective approach for calculating the tie-line ATC for longitudinal power systems.

\section{References}

[1] Takahashi R, Hiraki Y, Iwamoto S, et al. An efficient method of calculation for transient stability ATC in longitudinal power systems. In: Proc. of IEEE PES Power \& Energy Society General Meeting, 2009:1-6

[2] Iwamoto S. A formulation and a solution of transient stability problem using energy function. Electrical Engineering in Japan, 1985; 105(6):109-116.

[3] Ando R, Iwamoto S. Highly reliable transient stability solution method using energy type function. Trans. of IEE of Japan B, 108,253,(1988-6)

[4] Tanaka K. Fast Transient stability assessment based on energy function method: cooperation with simulation method. Research report. CRIEPI Komae Research Laboratory, No.T99019, 2000, (in Japanese).

[5] Nagata M, Tanaka K. A hybrid approach of contingency screening for ATC assessment with stability constraints. In: Proc. of CIGRE/IEEE PES International Symposium on Quality and Security of Electric Power Delivery Systems, 2003:137-142

[6] Anthony N, Michel AA, Fouad VV. Power system transient stability using individual machine energy functions. IEEE Trans. on circuits and systems, 1983; 30(5):266-276.

[7] The technical committee of the Institute of Electrical Engineers in Japan (IEEJ). Japanese power system models. [Online]. Available: http://www.iee.or.jp/pes/model/english/index.html 